\title{
Rare Giant Goronary Sinus Aneurysm with Aortic Dissection: A Case Report
}

\author{
Jing Ma, PhD ${ }^{a, 1}$, Tao Ma, MD ${ }^{b, 1}$,Jianmin Lu, MD ${ }^{a}$, Xiangke Ma, PhD ${ }^{c, *}$ \\ ${ }^{a}$ Department of Echocardiography, Shanghai Xuhui Central Hospital, Zhongshan-Xuhui Hospital, Fudan University, Shanghai, China; \\ ${ }^{b}$ Changzhi Medical College, Jiefangdong Road 161, Changzhi, China; ${ }^{c}$ Department of Neurosurgery, Beijing Chaoyang Hospital, \\ Capital Medical University, Jingyuan Road 5, Beijing, China \\ Received January 2, 2019; revision received June 19; accepted July 14.
}

\begin{abstract}
Aortic sinus aneurysm is a rare congenital malformation. During the embryonic period, dysplasia occurs in the middle elastic fiber of the aortic sinus wall and insufficient fusion occurs in the aortic valve annulus. Long-term high blood pressure can damage these weak compositions, leading to possible rupture and dissection of sinus aneurysms under a certain extreme hypertension. We report a rare case of giant coronary sinus aneurysm with intimal tear and aortic dissection that was detected by using transthoracic and transesophageal echocardiography.
\end{abstract}

Key words: Dissection; Goronary sinus aneurysm rupture; Hypertension

Advanced Ultrasound in Diagnosis and Therapy 2020;00:024-025

DOI: 10.37015/AUDT.2020.190031

$\mathrm{A}$ ortic sinus aneurysm is a rare cardiac anomaly that can be congenital or acquired. This cardiac defect links to connective and hypertension. This disease may present chest pain, and even sudden cardiac death, so prompt diagnosis and surgery is critical.

\section{Case presentation}

A 40-year-old male patient was admitted with sudden chest tightness and chest pain for two hours. Previous history included hypertension for 10 years with maximum of 190/100 mmHg. Physical examination shown that body temperature was $36.8^{\circ} \mathrm{C}$, sinus heart rate $92 \mathrm{bpm}$, respiration rate $23 \mathrm{bpm}$, and blood pressure $70 / 50 \mathrm{mmHg}$. The patient's expression was apathetic, neck was flexible, breathing sounds were coarse in both lungs, dry and moist rale were not found, the border of cardiac dullness expanded to the bottom left, heart rate was regular, heart sound was low and dull, diastolic murmur was heard at the left margin of the sternum of the third intercostal, and abdominal palpation was soft without tenderness and rebound tenderness. The lower limbs were swollen, limb muscle tension was normal, limb muscle strength grade 5, and a bilateral Pakistan's sign was negative.

Laboratory findings for myocardial infarction test was as follows: CK-MB $1.31 \mathrm{ng} / \mathrm{ml}$, cTnI $0.098 \mathrm{ng} / \mathrm{ml}$, MYO $46.63 \mathrm{ng} / \mathrm{ml} ; \mathrm{K}^{+} 3.5 \mathrm{mmol} / \mathrm{L}, \mathrm{Na}^{+}$ $135 \mathrm{mmol} / \mathrm{L}$, and Glu $5.34 \mathrm{mmol} / \mathrm{L}$. ECG examination showed sinus tachycardia type-B pre-excitation at 14:24 and sinus tachycardia type-B pre-excitation with STsegment elevation (elevation in leads V1-3) at 15:15.

\section{Echocardiographic examination}

Preoperative transthoracic echocardiography showed that the aorta had trefoil valves, which were mildly thickened and had good opening function, but when it closed, cracks in the valves were visible with a small amount of diastolic reflux and the aortic sinus widened to $10.10 \mathrm{~cm}$. The right coronary sinus aneurysm was seen as containinga giant spherical cyst approximately 9.94 $\times 7.95 \mathrm{~cm}$ in size. In addition, an intimal tear that was clearly observed by the transesophageal echocardiographic approach was interlinked with the giant aneurysm and the aorta. The giant aneurysm filled with cloudy echogenicity,

\footnotetext{
${ }^{1}$ Contributed equally

* Correspondence Author: Department of Neurosurgery, Beijing Chaoyang Hospital, Capital Medical University, Jingyuan Road 5, Beijing 100043, China

e-mail: maxiangke08@163.com unrestricted use, distribution and reproduction in any medium provided that the original work is properly attributed.
} 
and the right atrium and ventricles were severely pushed to the right rear by the cystic aneurysm lesion. A large pericardial effusion with blood clots was observed. The real time imaging of the heart showed that the right ventricle wall, interventricular septum, and left ventricular wall motion were hypokinetic. Ejection fraction (EF) of the left ventricular was $52 \%$.

\section{Surgical and pathologic findings}

The patient had an emergency operation. Surgical findings revealed a giant right coronary sinus cystic lesion $(10 \times 8 \mathrm{~cm})$ oppressed to the right atrium and right ventricle corresponding with the coronary sinus aneurysm. The intimal of the aneurysm was torn and extended into the aortic root, right coronary artery, and ascending aorta, which corresponded with aortic dissection. A large chocolate-colored thrombosis was observed within the aneurysm while blood effusion was observed in the pericardial cavity consisting of approximately $500 \mathrm{ml}$ blood with large blood clots. The left coronary sinus and non-normal coronary sinus had a normal appearance. As surgical result, this patient had coronary sinus aneurysm resection, and suture repair with replacement of artificial blood vessels. The pathological diagnosis was giant aortic sinus aneurysm with an intimal tear that extended into aortic root and ascending aorta. Proximal right coronary artery occlusion and pericardial tamponade were present.

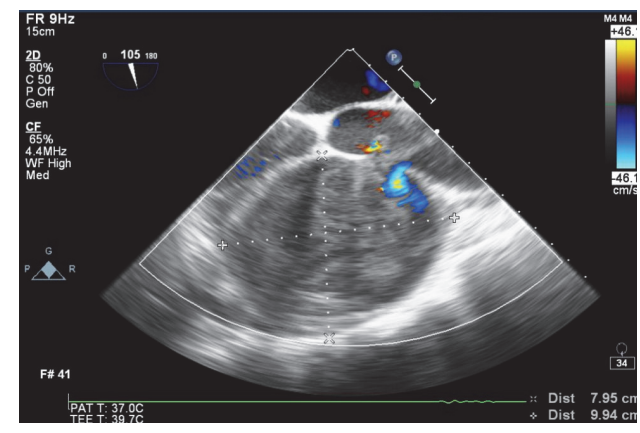

Figure 1 Giant coronary sinus aneurysm

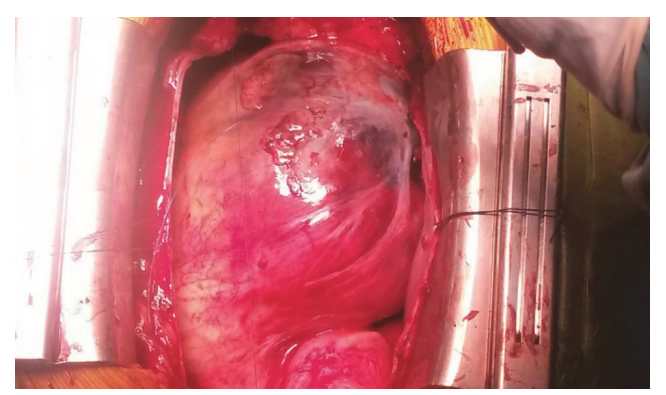

Figure 2 Intraoperative findings

\section{Discussion}

Due to the chronic hypertension, an aortic sinus aneurysm can gradually expand and result an intimal tear [1-3]. The area of tearing and rupture could be involved with the aortic root, the ascending aorta, and right coronary artery, which would cause blood to infiltrate the pericardium, and ultimately may result in cardiac tamponade. Because a ruptured giant sinus aneurysm can lead to pericardial tamponade, it is critical to give the patient emergency treatment immediately. However, this kind of aortic sinus lesion with the aortic root and ascending aorta dissection can be missed and misdiagnosed prior to surgical procedure [4,5], particularly because the preoperative transthoracic echocardiography might not easily and clearly detect it. Reasons that this aneurysm with dissection can be difficult to detect could include: (1) In obese patients, transthoracic ultrasound imaging in the aortic area may not be optimal in quality for distinguishing the dissection easily; (2) the patient may be experiencing early symptoms of shock and be in a critical condition, and thus the time for an ultrasound examine would short and limited.

\section{Conclusion}

This case study demonstrates the important role that intraoperative transesophageal echocardiography can be utilized in detecting these lesions and guiding the surgical procedure.

\section{Funding}

This work was supported by the National Natural Science Foundation of China (81501483), Shanghai Natural Science Foundation (18ZR1435400) and Major projects of Xuhui District Health and Family Planning Commission (SKLNDD-KF-201804).

\section{Conflicts of Interest}

The authors have declared no conflicts of interest.

\section{References}

[1] Agrawal Y, Chandrashekhar R, Pratt JW, Cole MD, Kamath S, Kalavakunta JK. Ruptured Sinus of Valsalva Aneurysm into the Left Atrium with Multiple Fistulous Communications: A Rare Cause of Heart Failure. Case Rep Cardiol 2015;2015:627946.

[2] Brabham KR, Roberts WC. Fatal intrapericardial rupture of sinus of Valsalva aneurysm. Am Heart J 1990;120:1455-1456.

[3] Jugpal TS, Dixit R, Lohchab S, Garg A. Giant Unruptured Sinus of Valsalva Aneurysm: An Unusual Cause of Right Heart Failure. J Clin Imaging Sci 2015;5:64.

[4] Morais H, Martins T. Right Sinus of Valsalva Aneurysm with Rupture into the Interventricular Septum and into the Left Ventricle. Heart Views 2015;16:114-115.

[5] Zhang J, Liu Y, Liu L, Deng Y. An extracardiac unruptured right sinus of valsalva aneurysm complicated with atherothrombosis. Echo Res Pract 2016;3:K1-K6. 